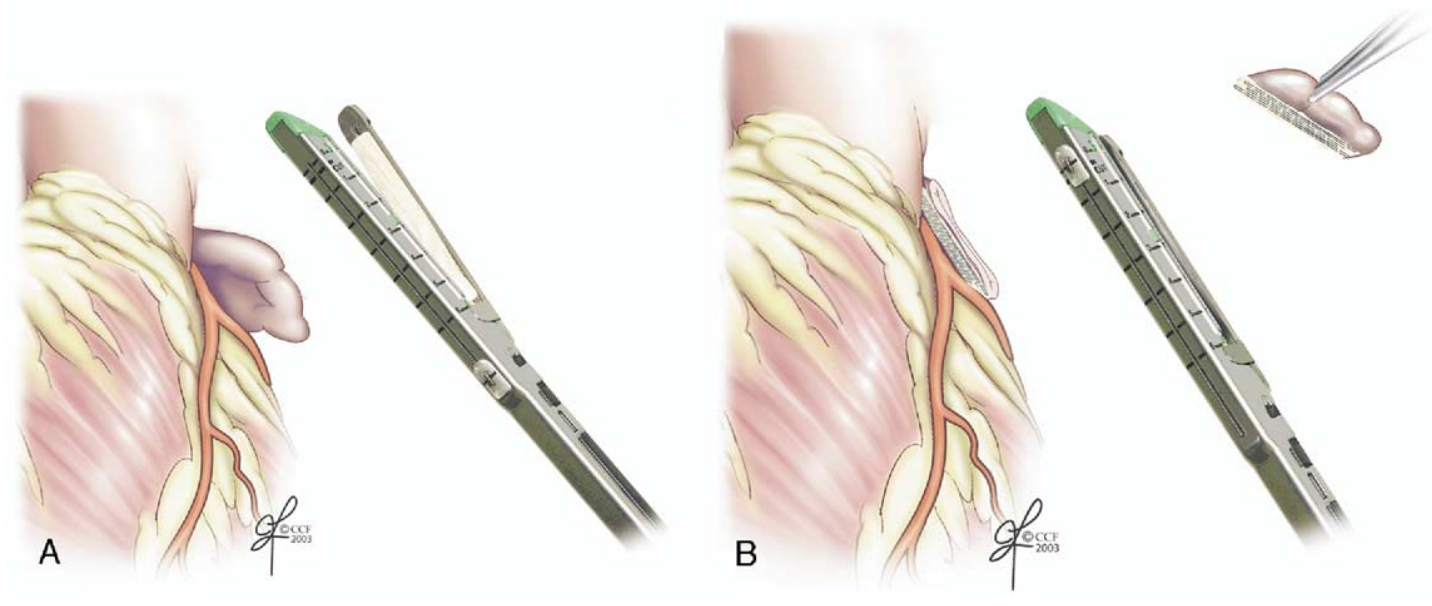

Figure 1. Stapled excision of LAA. A, Stapler is loaded with pericardial strips. B, LAA is excised, and area beneath the buttressed staple line is examined. Published with the permission of The Cleveland Clinic Foundation.

\section{References}

1. Johnson WD, Ganjoo AK, Stone CD, Srivyas RC, Howard M. The left atrial appendage: our most lethal human attachment! Surgical implications. Eur J Cardiothorac Surg. 2000;17:718-22.

2. Garcia-Fernandez MA, Perez-David E, Quiles J, Peralta J, Garcia-Rojas I, Bermejo J, et al. Role of left atrial appendage obliteration in stroke reduction in patients with mitral valve prosthesis. J Am Coll Cardiol. 2003;42:1253-8.
3. Rosenzweig BP, Katz E, Kort S, Schloss M, Kronzon I. Thromboembolism from a ligated left atrial appendage. $J$ Am Soc Echocardiogr. 2001;14:396-8.

4. Blackshear JL, Odell JA. Appendage obliteration to reduce stroke in cardiac surgical patients with atrial fibrillation. Ann Thorac Surg. 1996; 61:755-9.

5. DiSesa VJ, Tam S, Cohn LH. Ligation of the left atrial appendage using an automatic surgical stapler. Ann Thorac Surg. $1988 ; 46: 652-3$

\title{
Acute mitral regurgitation caused by papillary muscle rupture in the immediate postpartum period revealing Ehlers-Danlos syndrome type IV
}

Pascal Sève, MD, ${ }^{a}$ Olivier Dubreuil, MD, ${ }^{c}$ Fadi Farhat, MD, ${ }^{d}$ Henry Plauchu, MD, ${ }^{b}$ Paul Touboul, MD, ${ }^{\mathrm{c}}$ and Christiane Broussolle, MD, ${ }^{a}$ Lyon, France

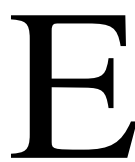

hlers-Danlos syndrome (EDS) type IV, the vascular type, is a rare autosomal dominant inherited disorder of connective tissue resulting from mutation of the COL $3 A 1$ gene encoding type III collagen. ${ }^{1-3}$ Affected patients often have life-threatening cardiovascular complications. ${ }^{1-3}$ We report here a

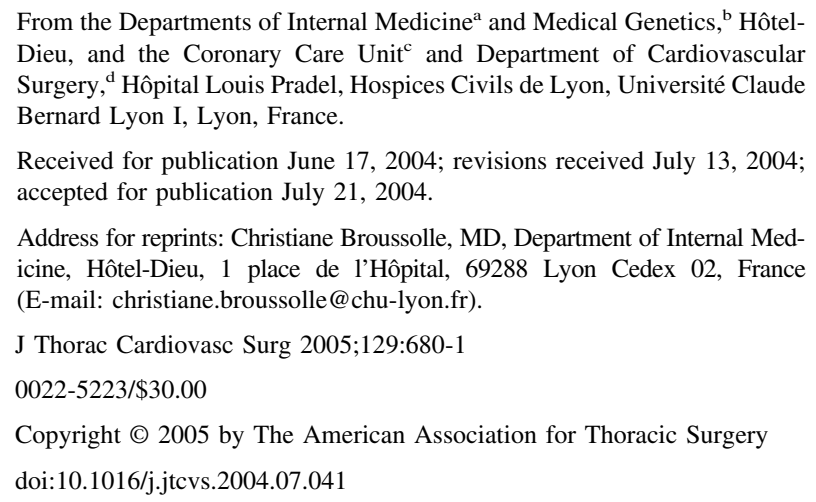

case of EDS type IV diagnosed 1 week after delivery in a patient with a severe mitral regurgitation caused by papillary muscle rupture requiring emergency surgical intervention.

\section{Clinical Summary}

A 36-year-old female patient was admitted to the intensive care unit with a diagnosis of severe mitral regurgitation caused by papillary muscle rupture. She had a medical history of pneumothorax at birth. One week before, the patient had been delivered spontaneously of her first pregnancy at 37 weeks' gestation. Delivery was complicated by vaginal bleeding from severe perineal tears. Five days later, the patient had respiratory distress with circulatory collapse. Chest radiography showed pulmonary edema. Transesophageal echocardiography revealed an acute mitral regurgitation with posterior leaflet mitral valve prolapse caused by papillary muscle rupture. The surgeon confirmed the diagnosis (Figure 1) and repaired the mitral valve by valvuloplasty after posterior leaflet mitral valve resection. Even though the tissues were friable, the surgical procedure did not pose technical diffi- 


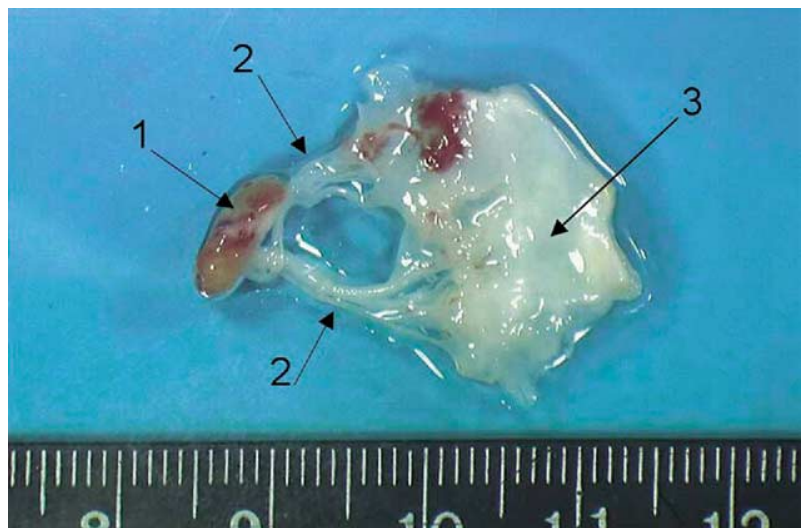

Figure 1. Papillary muscle rupture specimen includes papillary muscle (1), chordae tendineae (2), and posterior leaflet mitral valve (3).

culties. In the postoperative period there was no excessive bleeding, and skin cicatrization was achieved within normal times.

A diagnosis of EDS was suggested. The patient described easy bruising and abnormal scar formation. She had a facial dysmorphism with slenderness, prominent bones, hollow cheeks, lobeless ears, protruding eyes, pinched nose, and thin lips (Figure 2). Skin was thin and translucent, with visible veins. The skin on the extremities appeared aged. There was no skin hyperextensibility or joint hypermobility. Molecular studies by heteroduplex analysis of the COL3A1 complementary DNA obtained from cultured skin fibroblasts demonstrated a nonsense mutation in nucleotide 322 , resulting in the substitution of glycine for glutamate, confirming the diagnosis of EDS type IV. ${ }^{3}$

\section{Discussion}

Approximately $10 \%$ of patients with EDS have the type IV variant. $^{2}$ Because of the potential for life-threatening cardiovascular complications, this type is of particular concern to cardiovascular surgeons.

The clinical diagnosis is based on four major criteria: thin, translucent skin; arterial, intestinal, or uterine fragility or rupture; extensive bruising; an characteristic facial appearance. ${ }^{1}$ Minor diagnostic criteria include acrogeria, hypermobility of small joints, tendon and muscle rupture, talipes equinovarus, early-onset varicose veins, arteriovenous carotid-cavernous sinus fistula, pneumothorax or pneumohemothorax, gingival recession, positive family history, and sudden death in one or more close relatives. ${ }^{1}$ The presence of any two of the major criteria is highly indicative of the diagnosis. ${ }^{1}$ Our patient had three major and two minor diagnostic criteria. As in our case, diagnosis is confirmed by the identification of a mutation in the COL $3 A 1$ gene or by the demonstration that cultured fibroblasts synthesize abnormal type III procollagen molecules. $^{2}$

Vascular EDS results in premature death. The median survival of a cohort of 220 patients reported on by Pepin and colleagues ${ }^{2}$ was 48 years. Most deaths result from arterial rupture, with intestinal perforation being less frequently fatal. ${ }^{2,3}$ Arterial rupture is unpredictable, and surgical repair is difficult because of tissue

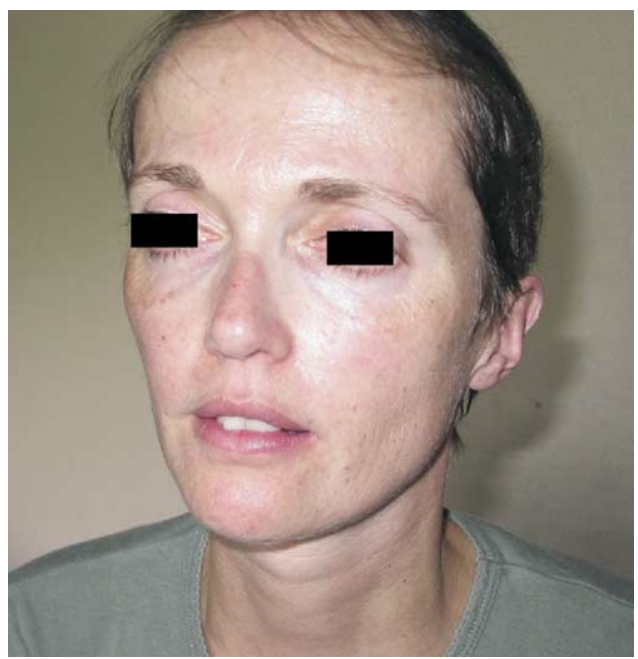

Figure 2. Facial appearance of the patient.

fragility. Arterial dissection, spontaneous arteriovenous fistulas, and aneurysm formation can also be observed, with a predilection for middle-sized arteries. ${ }^{3}$ Because arteriography often leads to extensive hematoma at puncture sites, minimally invasive techniques such as ultrasonography and magnetic resonance angiography should be used for diagnosis. ${ }^{3}$

Cardiac abnormalities, such as mitral valve prolapse and proximal aortic dilatation, are common features of types I through III EDS. ${ }^{1}$ In vascular EDS, marked mitral valve prolapse with severe mitral regurgitation has been previously reported, ${ }^{4}$ but acute mitral regurgitation caused by papillary muscle rupture has never before been described.

Pregnancy in women with vascular EDS increases the risk of arterial or uterine rupture, mainly during the last trimester. ${ }^{5}$ In the study reported by Pepin and colleagues, ${ }^{2}$ complications of pregnancy led to death in 12 of 81 women. On the basis of our observation, severe mitral regurgitation has to be added to the obstetric complications of vascular EDS.

Although no specific treatment is available in this syndrome, knowledge of the diagnosis may influence the management of surgery, pregnancy, genetic counseling, and major complications. Furthermore, genetic counseling with molecular diagnosis is recommended for family members of patients with EDS.

\section{References}

1. Beighton P, De Paepe A, Steinmann B, Tsipouras P, Wenstrup RJ. Ehlers-Danlos syndromes: revised nosology, Villefranche, 1997. Am J Med Genet. 1998;77:31-7.

2. Pepin M, Schwarze U, Superti-Furga A, Byers PH. Clinical and genetic features of Ehlers-Danlos syndrome type IV, the vascular type. $N$ Engl J Med. 2000;342:673-80.

3. Germain DP. Clinical and genetic features of vascular Ehlers-Danlos syndrome. Ann Vasc Surg. 2002;16:391-7.

4. Watanabe S, Ishimitsu T, Inoue K, Tomizawa T, Noguchi Y, Sugishita Y, et al. Type IV Ehlers-Danlos syndrome associated with mitral valve prolapse: a case report [in Japanese]. J Cardiol Suppl. 1988;18:97-106.

5. Lind J, Wallenburg HC. Pregnancy and the Ehlers-Danlos syndrome: a retrospective study in a Dutch population. Acta Obstet Gynecol Scand. 2002;81:293-300. 